Appl. Set-Valued Anal. Optim. 2 (2020), No. 3, pp. 295-303

Available online at http://asvao.biemdas.com

https://doi.org/10.23952/asvao.2.2020.3.04

\title{
THE ULAM-HYERS STABILITY OF AN ORDINARY DIFFERENTIAL EQUATION VIA GRONWALL LEMMAS
}

\author{
ADRIAN PETRUŞEL ${ }^{1,2}$, IOAN A. RUS $^{1, *}$ \\ ${ }^{1}$ Department of Mathematics, Babeş-Bolyai University Cluj-Napoca, Romania \\ ${ }^{2}$ Academy of Romanian Scientists, Bucharest, Romania
}

\begin{abstract}
Following [I.A. Rus, Gronwall lemma approach to the Hyers-Ulam-Rassias stability of an integral equation, in: Nonlinear Analysis and Variational Problems, Panos Pardalos, Themistocles M. Rassias, Akhtar A. Khan (ed.), pp. 147-152, Springer, 2009], we study the Ulam-Hyers stability of some ordinary differential equations based on Gronwall lemmas.
\end{abstract}

Keywords. Cauchy problem; Differential equation; Integral equation; Gronwall lemma; Ulam-Hyers stability.

\section{INTRODUCTION}

For the study of different Ulam types stability, there are various techniques in the literatures: exact solution approaches, fixed point approaches (weakly Picard operators,...), Gronwall's lemma approach, and so on. For the details on these approaches, we refer to [6, 8, 14, 15, $17,18,19]$ and the references therein. In this paper, based on the results presented in [14] and [17], we study the Ulam-Hyers stability of some ordinary differential equations by Gronwall lemma techniques.

\section{PRELIMINARIES}

2.1. Basic notions in the Ulam-Hyers stability of differential equations. Let $(\mathbb{B},|\cdot|)$ be a Banach space. Let $\varepsilon>0, a \in \mathbb{R}$, and $b \in \mathbb{R} \cup\{+\infty\}$ with $a<b$. We denote by $I$ an interval of the real axis $(I:=[a, b]$ or $[a, b[$, depending on the context).

Let $f \in C(I \times \mathbb{B}, \mathbb{B})$ be a given continuous operator. We consider the following problems:

1) the differential equation

$$
x^{\prime}(t)=f(t, x(t)), t \in I
$$

with its solution set denoted by $S_{0}$;

2) the differential inequation

$$
\left|x^{\prime}(t)-f(t, x(t))\right| \leq \varepsilon, t \in I,
$$

with the solution set (for each $\varepsilon>0$ ) denoted by $S_{\varepsilon}$. Notice that a function $y \in C^{1}(I, \mathbb{B}$ ) is a solution of (2.2) if and only if there exists a function $g \in C(I, \mathbb{B})$ (which may depend on $y$ ) such that

${ }^{*}$ Corresponding author.

E-mail addresses: petrusel@math.ubbcluj.ro (A. Petruşel), iarus@math.ubbcluj.ro (I.A. Rus).

Received September 14, 2020; Accepted October 21, 2020.

(C)2020 Applied Set-Valued Analysis and Optimization 
(i) $|g(t)| \leq \varepsilon$, for all $t \in I$;

(ii) $y^{\prime}(t)=f(t, y(t))+g(t)$, for every $t \in I$.

A solution to inequation (2.2) is called an $\varepsilon$-solution of equation (2.1).

The following concepts are important for our study in this paper (see [10, 18]).

Definition 2.1. The differential equation (2.1) is said to be Ulam-Hyers stable if there exists a real number $C_{f}>0$ such that, for each $\varepsilon>0$, there exists a retraction $r_{\varepsilon}: S_{\varepsilon} \rightarrow S_{0}$ satisfying

$$
\left|y(t)-r_{\varepsilon}(y)(t)\right| \leq C_{f} \varepsilon \text {, for each solution } y \in C^{1}(I, \mathbb{B}) \text { of (2.2) and every } t \in I \text {. }
$$

Definition 2.2. Differential equation (2.1) is said to be generalized Ulam-Hyers stable if there exists a function $\theta_{f} \in C\left(\mathbb{R}_{+}, \mathbb{R}_{+}\right)$with $\theta(0)=0$ such that, for each $\varepsilon>0$, there exists a retraction $r_{\varepsilon}: S_{\varepsilon} \rightarrow S_{0}$ satisfying

$$
\left|y(t)-r_{\varepsilon}(y)(t)\right| \leq \theta_{f}(\varepsilon) \text {, for each solution } y \in C^{1}(I, \mathbb{B}) \text { of (2.2) and every } t \in I .
$$

2.2. Gronwall lemmas. In this paper, we need the following Gronwall lemmas (see [2, 3, 4, $5,7,12,13,14])$.

Lemma 2.1. (Gronwall Lemma I.) Let $J$ be an interval of the real axis, $t_{0} \in J$ and the functions $h, k, u \in C\left(J, \mathbb{R}_{+}\right)$. If

$$
u(t) \leq h(t)+\left|\int_{t_{0}}^{t} k(s) u(s) d s\right|, \text { for all } t \in J
$$

then

$$
u(t) \leq h(t)+\left|\int_{t_{0}}^{t} h(s) k(s) e^{\left|\int_{s}^{t} k(\sigma) d \sigma\right|} d s\right|, \text { for all } t \in J .
$$

If, in addition, $h$ is increasing, then $(J:=[a, b[)$

$$
u(t) \leq h(t) e^{\int_{a}^{t} k(s) d s}, t \in[a, b[.
$$

Lemma 2.2. (Gronwall Lemma II.) Let $h \in C\left([a, b], \mathbb{R}_{+}\right)$and $\beta>0$ with $\beta(b-a)<1$. If $u \in C\left([a, b], \mathbb{R}_{+}\right)$satisfies the relation

$$
u(t) \leq h(t)+\beta \int_{a}^{b} u(s) d s, \text { for } t \in[a, b],
$$

then

$$
u(t) \leq h(t)+\frac{\beta}{1-\beta(b-a)} \int_{a}^{b} h(s) d s, \text { for every } t \in[a, b]
$$

3. AN HEURISTIC POINT OF VIEW ON THE ULAM-HYERS STABILITY OF A DIFFERENTIAL EQUATION

Let $f \in C([a, b] \times \mathbb{B}, \mathbb{B}), g \in C([a, b], \mathbb{B})$ and $\varepsilon \in \mathbb{R}_{+}^{*}$.

We consider the differential equations

$$
\begin{gathered}
x^{\prime}(t)=f(t, x(t)), t \in[a, b], \\
x^{\prime}(t)=f(t, x(t))+g(t), t \in[a, b],
\end{gathered}
$$

and the differential inequation

$$
\left|x^{\prime}(t)-f(t, x(t))\right| \leq \varepsilon, t \in[a, b] .
$$


For a function $x \in C^{1}([a, b], \mathbb{B})$ and for given $x_{0} \in \mathbb{B}$, we consider the Cauchy condition

$$
x(a)=x_{0} .
$$

We suppose that (see $[2,4,7,9,12])$ :

$(C)$ For each $x_{0} \in \mathbb{B}$, Cauchy problem (3.1)+(3.4) has a unique solution, denoted by $x\left(\cdot, x_{0}\right) \in$ $C^{1}([a, b], \mathbb{B})$;

$(P C)$ For each $g \in C([a, b], \mathbb{B})$ and each $x_{0} \in \mathbb{B}$, Cauchy problem (3.2)+(3.4) has a unique solution, denoted by $x\left(\cdot, x_{0}, g\right) \in C^{1}([a, b], \mathbb{B})$.

Let us consider the following subsets of $C^{1}([a, b], \mathbb{B})$ :

$$
\begin{aligned}
& S_{0}:=\left\{x\left(\cdot, x_{0}\right) \mid x_{0} \in \mathbb{B}\right\}, \\
& S_{\varepsilon}:=\left\{x\left(\cdot ; x_{0}, g\right)|g \in C([a, b], \mathbb{B}),| g(t) \mid \leq \varepsilon, \forall t \in[a, b], x_{0} \in \mathbb{B}\right\} .
\end{aligned}
$$

It is clear that, in the conditions $(C)$ and $(P C)$,

1) $S_{0}$ is the solution set of differential equation (3.1);

2) $S_{\varepsilon}$ is the solution set of differential inequation (3.3).

Let $y \in C^{1}([a, b], \mathbb{R})$ be a solution of (3.3). Then, there exists $g \in C^{1}([a, b], \mathbb{B})$ with $|g(t)| \leq \varepsilon$, $\forall t \in[a, b]$, such that $y$ is a solution of differential equation (3.2). Let $x(\cdot, y(a))$ be the unique solution to (3.1) with $x(a)=y(a)$.

For $y$ and $x(\cdot, y(a))$, we have

$$
y(t)=y(a)+\int_{a}^{t} f(s, y(s)) d s+\int_{a}^{t} g(s) d s, t \in[a, b],
$$

and

$$
x(t ; y(a))=y(a)+\int_{a}^{t} f(s, x(s ; y(a)) d s, t \in[a, b] .
$$

The above relations imply that

$$
|y(t)-x(t ; y(a))| \leq \int_{a}^{t}|f(s, y(s))-f(s, x(s ; y(a)))| d s+(b-a) \varepsilon, t \in[a, b] .
$$

In some conditions on $f$, we can obtain estimations for $|y(t)-x(t ; y(a))|$. For example, let us suppose that

(Lip) There exists $L>0$ such that

$$
|f(t, u)-f(t, v)| \leq L|u-v|, \forall t \in[a, b], \forall u, v \in \mathbb{B} .
$$

With this condition, we have that

$$
|y(t)-x(t ; y(a))| \leq L \int_{a}^{t}|y(s)-x(s ; y(a))| d s+(b-a) \varepsilon, t \in[a, b] .
$$

From Gronwall Lemma I (see Lemma 2.1), the estimate follows

$$
|y(t)-x(t ; y(a))| \leq(b-a) e^{L(b-a)} \varepsilon, t \in[a, b] .
$$

From the above considerations, for each $\varepsilon>0$, we have a retraction, $r_{\varepsilon}: S_{\varepsilon} \rightarrow S_{0}$, defined by $y \mapsto x(\cdot, y(a))$, for which

$$
\left|y(t)-r_{\varepsilon}(y)(t)\right| \leq c \varepsilon, t \in[a, b]
$$

where $c=(b-a) e^{L(b-a)}$.

Since condition (Lip) implies the conditions $(C)$ and $(P C)$, we have the following result. 
Theorem 3.1. Let us consider the differential equation (3.1). Suppose that the condition (Lip) holds. Then, for each $\varepsilon>0$, there exists a retraction $r_{\varepsilon}: S_{\varepsilon} \rightarrow S_{0}$ such that

$$
\left|y(t)-r_{\varepsilon}(y)(t)\right| \leq(b-a) e^{L(b-a)} \varepsilon, \forall y \in S_{\varepsilon} \text { and } \forall t \in[a, b],
$$

i.e., equation (3.1) is Ulam-Hyers stable.

Remark 3.1. Let us take $\mathbb{B}:=\mathbb{R}^{m}$ and denote a norm on $\mathbb{R}^{m}$ by $|\cdot|$. In this case, since all norms on $\mathbb{R}^{m}$ are equivalent, if equation (3.1) is Ulam-Hyers stable with respect to a norm on $\mathbb{R}^{m}$, then it is Ulam-Hyers stable with respect to each other norm on $\mathbb{R}^{m}$ (see $[11,16,18]$ ). If we denote by $L_{|\cdot|}$, the Lipschitz constant of $f$ with respect to $|\cdot|$, then we have, in terms of the norm $|\cdot|$, the following estimation

$$
\left|y(t)-r_{\varepsilon}(y)(t)\right| \leq(b-a) e^{L_{|\cdot|}(b-a)} \varepsilon, \text { for all } y \in S_{\varepsilon} \text { and } t \in[a, b] .
$$

For example, in the case that $f(t, y(t))=A(t) x(t)$, where $A \in C\left([a, b], \mathbb{R}^{m \times m}\right.$ is a matrix function and $|A(t)|$ is the corresponding matrix norm of $A(t)$ with respect to the vector norm $|\cdot|$, we have

$$
L_{|\cdot|}=\max _{t \in[a, b]}|A(t)|
$$

From Theorem 3.1, we have the following.

Theorem 3.2. If $A \in C\left([a, b], \mathbb{R}^{m \times m}\right)$, then the linear system of differential equations

$$
x^{\prime}(t)=A(t) x(t), t \in[a, b],
$$

is Ulam-Hyers stable.

We have a similar result in the case $\mathbb{B}:=\mathbb{C}^{m}$.

Remark 3.2. Let $(\mathbb{B},|\cdot|):=\left(l_{2}(\mathbb{R}),|\cdot|_{2}\right)$. In this case, equation (3.1) is a countable system of differential equations (see [20]). In the corresponding (Lip) condition, this system is UlamHyers stable.

Remark 3.3. Instead of condition (Lip), some authors (see, for example, [6]) considered the condition

$$
(\operatorname{Lip}(t)) \quad|f(t, u(t))-f(t, v(t))| \leq L|u(t)-v(t)| \text {, for every } u, v \in C([a, b], \mathbb{B}), t \in[a, b] .
$$

It is clear that conditions $(\operatorname{Lip})$ and $(\operatorname{Lip}(t))$ are equivalent.

\section{SECOND ORDER DIFFERENTIAL EQUATIONS}

For a better understanding of the Ulam-Hyers stability of $n$-order differential equations, we consider, for simplicity, the following second order differential equation

$$
-x^{\prime \prime}(t)=f(t, x(t)), t \in[a, b]
$$

where $f \in C([a, b] \times \mathbb{R})$.

For $g \in C[a, b]$, we consider the differential equation

$$
-x^{\prime \prime}(t)=f(t, x(t))+g(t), t \in[a, b],
$$

and for $\varepsilon>0$, the following differential inequation

$$
\left|-x^{\prime \prime}(t)-f(t, x(t))\right| \leq \varepsilon, t \in[a, b]
$$


Let us suppose that:

$(C)$ for all $x_{0}, x_{0}^{\prime} \in \mathbb{R}$, there exists a unique solution, denoted by $x\left(\cdot ; x_{0}, x_{0}^{\prime}\right) \in C^{2}[a, b]$ of $(4.1)$, such that

$$
x(a)=x_{0}, x^{\prime}(a)=x_{0}^{\prime},
$$

$(P C)$ for all $g \in C[a, b], x_{0}, x_{0}^{\prime} \in \mathbb{R}$, there exists a unique solution, denoted by $x\left(\cdot ; x_{0}, x_{0}^{\prime}, g\right) \in$ $C^{2}[a, b]$ of (4.2), such that

$$
x(a)=x_{0}, x^{\prime}(a)=x_{0}^{\prime}
$$

In conditions $(C)$ and $(P C)$,

$$
S_{0}:=\left\{x\left(\cdot ; x_{0}, x_{0}^{\prime}\right) \mid x_{0}, x_{0}^{\prime} \in \mathbb{R}\right\}
$$

is the solution set of (4.1) and

$$
S_{\varepsilon}:=\left\{x\left(\cdot ; x_{0}, x_{0}^{\prime}, g\right)\left|x_{0}, x_{0}^{\prime} \in \mathbb{R}, g \in C[a, b],\right| g(t) \mid \leq \varepsilon, \forall t \in[a, b]\right\}
$$

is the solution set of (4.3).

Now, let $y \in C^{1}[a, b]$ be a solution of (4.3). Then, there exists $g \in C[a, b],|g(t)| \leq \varepsilon, t \in[a, b]$, such that $y$ is a solution of (4.2). Let $x\left(\cdot ; y(a), y^{\prime}(a)\right)$ be the unique solution to (4.1). For $y$ and $x\left(\cdot ; y(a), y^{\prime}(a)\right)$, we have

$$
y(t)=y(a)+y^{\prime}(a)(t-a)+\int_{a}^{t}(s-t) f(s, y(s)) d s+\int_{a}^{t}(s-t) g(s) d s
$$

and

$$
x\left(t ; y(a), y^{\prime}(a)\right)=y(a)+y^{\prime}(a)(t-a)+\int_{a}^{t}(s-t) f\left(s, x\left(s ; y(a), y^{\prime}(a)\right)\right) d s .
$$

These imply that

$$
\left|y(t)-x\left(t ; y(a), y^{\prime}(a)\right)\right| \leq \int_{a}^{t}\left|f(s, y(s))-f\left(s, x\left(s ; y(a), y^{\prime}(a)\right)\right)\right|+\frac{1}{2}(b-a)^{2} \varepsilon .
$$

If we suppose, for example, the condition $(\operatorname{Lip})$ for $f$, then

$$
\left|y(t)-x\left(t ; y(a), y^{\prime}(a)\right)\right| \leq \frac{1}{2}(b-a)^{2} e^{L(b-a)} \varepsilon,
$$

for all $y \in S_{\varepsilon}$ and $t \in[a, b]$.

In the above conditions, we get the retractions

$$
r_{\varepsilon}: S_{\varepsilon} \rightarrow S_{0}, y \mapsto x\left(\cdot ; y(a), y^{\prime}(a)\right),
$$

with

$$
\left|y(t)-r_{\varepsilon}(y)(t)\right| \leq \frac{1}{2}(b-a)^{2} e^{L(b-a)} \varepsilon,
$$

for all $y \in S_{\varepsilon}$ and $t \in[a, b]$.

Thus, by the above observations, we have the following result.

Theorem 4.1. If condition (Lip) takes place, then differential equation (4.1) is Ulam-Hyers stable. 
Now, let us work in more restrictive conditions:

(B) for all $\alpha, \beta \in \mathbb{R}$, equation (4.1) has a unique solution, denoted by $x(\cdot ; \alpha, \beta) \in C^{2}[a, b]$, such that

$$
x(a)=\alpha, x(b)=\beta .
$$

$(P B)$ for all $g \in C[a, b]$ and all $\alpha, \beta \in \mathbb{R}$, the equation (4.2) has a unique solution, denoted by $x(\cdot ; \alpha, \beta, g) \in C^{2}[a, b]$, such that

$$
x(a)=\alpha, x(b)=\beta .
$$

In these conditions, we denote by

$$
S_{0}=\{x(\cdot, \alpha, \beta) \mid \alpha, \beta \in \mathbb{R}\}
$$

the solution set of (4.1) and by

$$
S_{\varepsilon}=\{x(\cdot, \alpha, \beta, g)|\alpha, \beta \in \mathbb{R}, g \in C[a, b],| g(t) \mid \leq \varepsilon, t \in[a, b]\}
$$

the solution set of (4.3).

We remark that the operator $\rho_{\varepsilon}: S_{\varepsilon} \rightarrow S_{0}$, defined by $y \mapsto x(\cdot ; y(a), y(b))$ is a retraction of $S_{\varepsilon}$ on $S_{0}$. Let us estimate $\left|y(t)-\rho_{\varepsilon}(y)(t)\right|$.

We have, in the terms of the Green function $G(s, t)$ of the operator $-x^{\prime \prime}$, the following relations for $y$ and $x(\cdot ; y(a), y(b))$,

$$
\begin{gathered}
y(t)=\frac{b-t}{b-a} y(a)+\frac{t-a}{b-a} y(b)+\int_{a}^{b} G(t, s) f(s, y(s)) d s+\int_{a}^{b} G(t, s) g(s) d s, \\
t \in[a, b]
\end{gathered}
$$

and

$$
\begin{gathered}
x(t ; y(a), y(b))=\frac{b-t}{b-a} y(a)+\frac{t-a}{b-a} y(b)+\int_{a}^{b} G(t, s) f(s, x(s ; y(a), y(b))) d s, \\
t \in[a, b] .
\end{gathered}
$$

Recall that the Green function of the operator $-x^{\prime \prime}$ is given by

$$
G(t, s)= \begin{cases}\frac{(s-a)(b-t)}{b-a} & \text { if } \quad s \leq t, \\ \frac{(t-a)(b-s)}{b-a} & \text { if } s \geq t .\end{cases}
$$

The above relations imply that

$$
\begin{gathered}
|y(t)-x(t ; y(a), y(b))| \leq \int_{a}^{b} G(t, s)|f(s, y(s))-f(s, x(s ; y(a), y(b)))| d s+\int_{a}^{b} G(t, s)|g(s)| d s \leq \\
\int_{a}^{b} G(t, s)|f(s, y(s))-f(s, x(s ; y(a), y(b)))|+\frac{(b-a)^{2}}{8} \varepsilon .
\end{gathered}
$$

If we suppose, for example, the condition ( $\mathrm{Lip})$ for $f$, then

$$
|y(t)-x(t ; y(a), y(b))| \leq L \int_{a}^{b} G(t, s)|y(s)-x(s ; y(a), y(b))| d s+\frac{(b-a)^{2}}{8} \varepsilon .
$$

for all $y \in S_{\varepsilon}$ and $t \in[a, b]$. Thus, by Gronwall Lemma II, (see Lemma 2.2), we get

$$
|y(t)-x(t ; y(a), y(b))| \leq \frac{(b-a)^{2}}{8} \varepsilon+\frac{(b-a)^{3}}{8} \varepsilon \frac{L \frac{b-a}{4}}{1-L \frac{(b-a)^{2}}{4}}=\frac{(b-a)^{2}}{8}\left(1+\frac{L(b-a)^{2}}{4-L(b-a)^{2}}\right) \varepsilon \text {. }
$$


In view of the above, we get the following result immediately.

Theorem 4.2. If condition (Lip) takes place and $\frac{L(b-a)^{2}}{4}<1$, then differential equation (4.1) is Ulam-Hyers stable.

Remark 4.1. For related results, we refer to [15, pp. 131-132].

Let us also notice that, in general, $r_{\varepsilon} \neq \rho_{\varepsilon}$, as we will see in the following example.

Example 4.1. Let us consider the differential equations

$$
-x^{\prime \prime}=-x, t \in[0,1]
$$

and

$$
-x^{\prime \prime}=-x+1, t \in[0,1] .
$$

Here $g(t)=1$. Then, for $\varepsilon \geq 1$, the function $y(t)=e^{t}+1$ is a solution of the differential inequation

$$
\left|-x^{\prime \prime}+x\right| \leq \varepsilon
$$

If we consider the initial conditions

$$
\left\{\begin{array}{l}
y(0)=2 \\
y^{\prime}(0)=1
\end{array}\right.
$$

then, by an easy calculation, we get

$$
x(t ; 2,1)=\frac{3}{2} e^{t}+\frac{1}{2} e^{-t}:=r_{\varepsilon}(y)(t) .
$$

On the other hand, if we consider the bilocal conditions

$$
\left\{\begin{array}{c}
y(0)=2 \\
y(1)=e+1
\end{array}\right.
$$

then

$$
x(t ; 2, e+1)=\frac{2 e^{-1}-e-1}{e^{-1}-e} e^{t}+\frac{1-e}{e^{-1}-e} e^{-t}:=\rho_{\varepsilon}(y)(t) .
$$

5. Differential equations With Solutions in $C^{1}([a,+\infty[, \mathbb{B})$

Let $f \in C\left(\left[a, \infty[, \times \mathbb{B}, \mathbb{B}), g \in C\left(\left[a,+\infty[, \mathbb{B}), \varepsilon>0\right.\right.\right.\right.$ and $\varphi \in C\left(\left[a,+\infty\left[, \mathbb{R}_{+}^{*}\right)\right.\right.$. We consider the differential equations

$$
\begin{gathered}
x^{\prime}(t)=f(t, x(t)), t \in[a,+\infty[ \\
x^{\prime}(t)=f(t, x(t))+g(t), t \in[a,+\infty[,
\end{gathered}
$$

and the differential inequations

$$
\begin{gathered}
\left|x^{\prime}(t)-f(t, x(t))\right| \leq \varepsilon, \forall t \in[a,+\infty[ \\
\left|x^{\prime}(t)-f(t, x(t)) \leq \varepsilon \varphi(t)\right|, \forall t \in[a,+\infty[,
\end{gathered}
$$

and

$$
\left|x^{\prime}(t)-f(t, x(t))\right| \leq \varphi(t), \forall t \in[a,+\infty[.
$$

As in Section 3, we suppose (see also $[1,21]$ ) that

$(C)$ for each $x_{0} \in \mathbb{B}$ the equation $(5.1)$ has a unique solution, denoted by $x\left(\cdot, x_{0}\right) \in C^{1}([a, \infty[, \mathbb{B})$, such that $x(a)=x_{0}$. 
$(P C)$ the equation (5.2) satisfies condition $(C)$ for each $g \in C([a,+\infty[, \mathbb{B})$.

Let $S_{0}$ be the solution set of (5.1), $S_{\varepsilon}, S_{\varepsilon, \varphi}, S_{\varphi}$ the solution set of (5.3), (5.4) and (5.5), respectively. As in Section 3, we consider the retractions

$$
r_{\varepsilon}: S_{\varepsilon} \rightarrow S_{0}, r_{\varepsilon, \varphi}: S_{\varepsilon, \varphi} \rightarrow S_{0}, r_{\varphi}: S_{\varphi} \rightarrow S_{0}
$$

For $r_{\varepsilon}$, we have the estimate

$$
\left|y(t)-r_{\varepsilon}(y)(t)\right| \leq(t-a) e^{L(t-a)} \varepsilon, \forall t \in[a,+\infty[.
$$

The problem is that this estimate is not of Ulam-Hyers type.

Moreover, by simple examples, we observe that, in general, the differential equations is not Ulam-Hyers stable $([8,15])$ on $[a,+\infty[$.

Example 5.1. We consider the case $\mathbb{B}:=\mathbb{R}$ and $f=0$.

In this case, we have

$$
x^{\prime}(t)=0, t \in[a,+\infty[
$$

and

$$
\left|x^{\prime}(t)\right| \leq \varepsilon, t \in[a, \infty[.
$$

The function $y(t)=t \varepsilon, t \in[a,+\infty[$ is a solution of (5.7) and all solutions of (5.6) are $x=c$, $c \in \mathbb{R}$. We remark that $|y(t)-c| \rightarrow \infty$ as $t \rightarrow \infty$.

The function $y(t)=\varepsilon \int_{a}^{t} \varphi(s) d s$ is a solution of

$$
\left|x^{\prime}(t)\right| \leq \varepsilon \varphi(t), \text { for } t \in[a,+\infty[.
$$

In this case,

$$
|y(t)-0|=\left|\varepsilon \int_{a}^{t} \varphi(t) d s\right|=\varepsilon \int_{a}^{t} \varphi(s) d s .
$$

To have an estimate of Ulam-Hyers-Rassias type, the function $\varphi$ must satisfy a strong restrictive condition (see $[8,15]$ ) of the following form:

(CC) there exists $\lambda>0$ such that $\int_{a}^{t} \varphi(s) d s \leq \lambda \varphi(t), \forall t \in[a,+\infty[$.

So, nothing remains but to estimate

$$
\left|y(t)-\rho_{\varepsilon, \varphi}(y)(t)\right|
$$

and

$$
\left|y(t)-\rho_{\varphi}(y)(t)\right|
$$

\section{REFERENCES}

[1] R.P. Agarwal, D. O’Regan, Infinite Interval Problems for Differential, Difference and Integral Equations, Kluwer Acad. Publ. 2001.

[2] H. Amann, Ordinary Differential Equations, Degruyter, Berlin, 1990.

[3] D. Bainov, P. Simeonov, Integral Inequalities and Applications, Springer, Netherlands, 1992.

[4] E.A. Coddington, N. Levinson, The Theory of Ordinary Differential Equations, McGraw-Hill, New York, 1955.

[5] J. Dieudonné, Foundations of Modern Analysis, Academic Press, New York, 1960.

[6] P. Găvrută, L. Găvruţă, A new method for the generalized Hyers-Ulam-Rassias stability, Int. J. Nonlinear Anal. Appl. 1 (2010), 11-18.

[7] P. Hartman, Ordinary Differential Equations, Wiley, New York, 1964. 
[8] S.-M. Jung, A fixed point approach to the stability of a Volterra integral equation, Fixed Point Theory Appl. 2007 (2007), Article ID 57064.

[9] J.P. La Salle, The Stability of Dynamical Systems, SIAM, Philadelphia, 1976.

[10] A. Petruşel, I.A. Rus, Ulam stability of zero point equations, In: Ulam Type Stability, J. Brzedek, D. Popa, Th.M. Rassias (eds.), pp. 345-364, Springer, 2019,.

[11] A. Petruşel, I.A. Rus, M.A. Şerban, The role of equivalent metrics in fixed point theory, Topological Methods in Nonlinear Anal. 41 (2013), 85-112.

[12] L.C. Piccinini, G. Stampacchia, G. Vidossich, Ordinary Differential Equations in $\mathbb{R}^{n}$, Springer, New York, 1984.

[13] I.A. Rus, Fixed points, upper and lower fixed points: abstract Gronwall lemmas, Carpathian J. Math. 20 (2004), 125-134.

[14] I.A. Rus, Gronwall lemma approach to the Hyers-Ulam-Rassias stability of an integral equation, In: Nonlinear Analysis and Variational Problems, P. Pardalos, Th.M. Rassias, A.A. Khan, (eds.), pp. 147-152, Springer, 2009.

[15] I.A. Rus, Ulam stability of ordinary differential equations, Studia Univ. Babeş-Bolyai Math. 54 (2009), 125133.

[16] I.A. Rus, Remarks on Ulam stability of the operatorial equations, Fixed Point Theory, 10 (2009), 305-320.

[17] I.A. Rus, Ulam stabilities of ordinary differential equations in a Banach space, Carpathian J. Math. 26 (2010), 103-107.

[18] I.A. Rus, Results and problems in Ulam stability of operatorial equations and inclusions, In: Handbook of Functional Equations: Stability Theory, Th. M. Rassias (ed.), pp. 323-352, Springer, 2014.

[19] I.A. Rus, A. Petruşel, G. Petruşel, Fixed Point Theory, Cluj Univ. Press, Cluj-Napoca, 2008.

[20] A.M. Samoilenko, Yu.V. Teplinskii, Countable System of Differential Equations, de Gruyter, Berlin, 2003.

[21] C. Vladimirescu, C. Avramescu, Applications of the Fixed Point Method to Ordinary Differential and Integral Equations on Noncompact Intervals, Universitaria Press, Craiova, 2006. 